\title{
Balanced Chromosomal Translocation of Chromosomes 6 and 7: A Rare Male Factor of Spontaneous Abortions
}

\author{
Sefa Resim¹, Ateş Kadıoğlu², Tolga Akman³ , Ayşe Gül Bayrak³, Erkan Efe \\ ${ }^{1}$ Department of Urology, Kahramanmaraş Sütçü Imam University Faculty of Medicine, Kahramanmaraş, Turkey \\ ${ }^{2}$ Department of Urology, İstanbul University Faculty of Medicine, İstanbul, Turkey \\ ${ }^{3}$ Department of Internal Diseases, İstanbul University Faculty of Medicine, İstanbul, Turkey
}

\begin{abstract}
Background: Carriers of structural chromosomal rearrangements such as Robertsonian or reciprocal translocations have an increased risk of spontaneous abortion and producing offspring with genetic abnormalities.

Case Report: We report a man with balanced chromosomal translocations located at 6p22, and 7q22. His wife has a history of four spontaneous abortions.

Conclusion: Couples with a history of abortions should be investigated cytogenetically, after other causes of miscarriages are excluded. The possibility of spontaneous abortions can be reduced with preimplantation genetic diagnosis (PGD) before embryo transfer.
\end{abstract}

Key Words: Balanced chromosomal translocation, spontaneous abortions, preimplantation genetic diagnosis

Received: 18.07.2011

Accepted: 30.10.2012

\section{Introduction}

Spontaneous abortions (SABs) occur due to causes such as uterine abnormalities, as well as immunological and genetic factors. Cytogenetic evaluation of the products of conception is often unhelpful, but may help to uncover any significant somatic anomalies in the male and/or female partner. Medical evaluation of couples with recurrent SABs should include karyotypic evaluation of both partners, according to Practice Committee guidelines, because approximately $50 \%$ of SABs stem from genetic abnormalities (1). The most common structural anomaly in infertility patients is sex chromosome aneuploidy. Balanced translocation is the most common structural chromosomal aberration in humans. Other chromosomal aberrations include deletion, fusion, and translocation. The reported incidence of balanced chromosomal translocations in couples with a history of SABs ranges from $3 \%$ to $6 \%$ (2). $\mathrm{SABs}$ are common, but recurrent $\mathrm{SABs}$ are more rare. In this report, we describe a couple (a man who presented with balanced chromosomal translocations on two chromosomes [6 and 7] and a woman with a history of four spontaneous abortions). Institutional review board approval was obtained to report the findings of the case.

\section{Case Report}

A couple who had conceived but the woman had had recurrent miscarriages for 4 years presented to our outpatient clinic (a 33-year-old husband and 20-year-old wife). The wife had had four spontaneous abortions during the first trimes- ter. Cytogenetic investigation had not been performed on the previous abortion materials. The husband's past history and family history were not contributory. The proband was phenotypically normal. The husband's physical examination revealed no abnormality except a reduced testicular volume (testicular volume was $17 \mathrm{~mL}$ for the right testicle and $15 \mathrm{~mL}$ for the left testicle). His wife had normal menstrual cycles, and her gynaecological evaluation revealed no abnormality. The patient underwent repeated semen analysis, karyotyping of peripheral blood lymphocytes, and molecular tests for Y-chromosome microdeletions. Repeated semen analysis showed asthenospermia and abnormality of sperm morphology. He had a normal sperm count (average $29.5 \times 105 / \mathrm{cc}$ ) (Table 1).

Chromosomal analysis was performed using three different multiplex PCR methods. Thirteen sequence-tagged sites (STS) on the Y chromosome were investigated (ZFX/ZFY, SRY, AZFa: sY84, sY86, DFFRY, DBY, AZFb: sY117, sY125, sY127, sY134, AZFc: DAZ gene- sY254, and sY255), and Y chromosome microdeletion was not detected.

Chromosomal analysis of peripheral blood lymphocytes was conducted using the standard methods. The proband proved to be a carrier of chromosomal translocation and his partner's karyotype was found to be normal. The karyotype of the proband was $46, \mathrm{XY}, \mathrm{t}(6 ; 7)(\mathrm{p} 22 ; \mathrm{q} 22)$ as seen in Figure 1.

\section{Analysis Method}

Standard in vitro lymphocyte culture was used for chromosomal analysis. Blood (2 mL) was drawn into a heparinised syringe. Twenty percent of fetal bovine serum (FBS) (PAA Laboratories $\mathrm{GmbH}$, Linz, Austria), 1\% penicillin-streptomycin 


\section{Table 1. The semen parameters of the patient}

\section{First Semen Analysis}

volume: $2.5 \mathrm{~mL}$

$\mathrm{pH}: 7.5$

sperm concentration: $33 \times 10^{6}$ spermatozoa $/ \mathrm{mL}$

total sperm number: $82.5 \times 10^{6}$ spermatozoa $/ \mathrm{mL}$ ejaculate

motility $(a+b): 11+16 \%$

morphology (Kruger's strict criteria): 5\%

WBCs: less than 1 million/mL

\section{Second Semen Analysis}

volume: $3 \mathrm{~mL}$

pH: 7.5

sperm concentration: $26 \times 10^{6}$ spermatozoa $/ \mathrm{mL}$

total sperm number: $78 \times 10^{6}$ spermatozoa $/ \mathrm{mL}$ ejaculate

motility $(a+b): 13+19 \%$

morphology (Kruger's strict criteria): $6 \%$

WBCs: less than 1 million/mL

(PAA Laboratories GmbH, Linz, Austria), 1\% L-glutamine (PAA Laboratories $\mathrm{GmbH}$, Linz, Austria), and 1.5\% of phytohaemagglutinin-M (PHA-M) (Biological Industries, Kibbutz Beit Haemek, Israel) were added to RPMI 1640 medium (Biochrom KG, Berlin, Germany) to prepare the culture medium. The blood sample obtained from the patient was added to the medium, which was incubated for 72 hours at $37^{\circ} \mathrm{C}$. At hour $70,0.04$ $\mathrm{ng} / \mathrm{mL}$ of colchicine (Colchicine powder, Sigma Chemical Co., St. Louis, NJ, USA) was added to the culture. Peripheral blood lymphocytes were harvested by standard procedures. The chromosomes were banded using the Giemsa-TrypsinGiemsa (GTG) banding technique, and twenty metaphases with a resolution of 500-550 bands were evaluated under a light microscope (3). The couple was referred to an in vitro fertilisation centre for intracytoplasmic sperm injection (ICSI) cycles and preimplantation genetic diagnosis (PGD).

\section{Discussion}

There is a causal relationship between chromosomal aberrations and male infertility. The incidence of chromosomal aberrations is commonly inversely correlated with sperm count. Chromosomal aberrations, such as deletion, fusion, and translocation, may cause structural alterations of the nucleic acid at the broken points and lead to genetic defects. Chromosomal translocations involve the transfer of genetic material from one chrosome to another, and can be reciprocal or Robertsonian. While the reciprocal translocations involve the breakage of two non-homologous chromosomes with exchange of segments, Robertsonian translocations involve breakpoints close to the centromere of two acrocentric chromosomes. The importance of translocations relates to the pattern of segregation at meiosis. The chances of conceiving a chromosomally

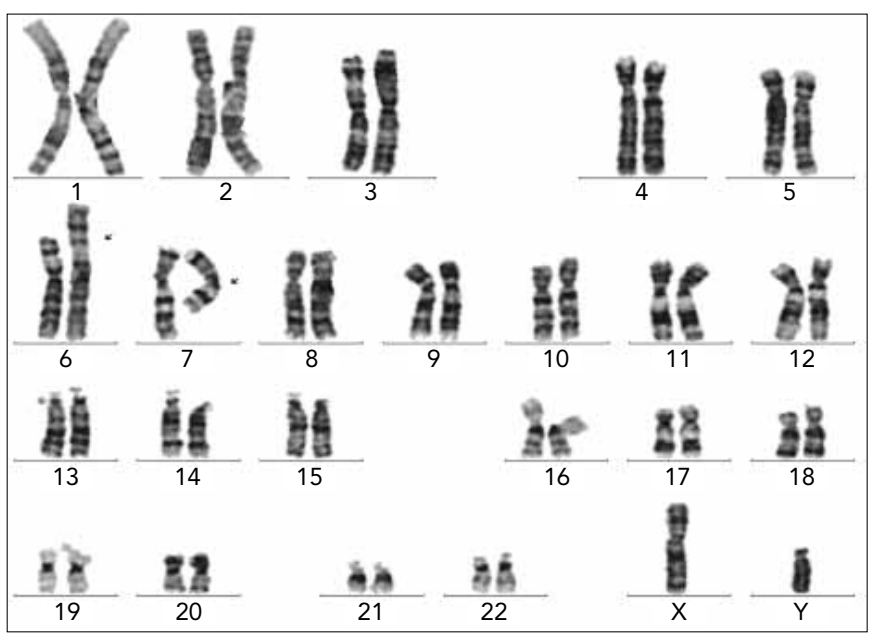

Figure 1. The karyotype of the husband

abnormal embryo are in the range of $20 \%$ to $80 \%$ for carriers of translocation, depending on the chromosomes involved in the translocations, the position of the breakpoints, and the sex of the translocation carrier (4).

Male infertility has been reported to be associated with chromosomal abnormalities that usually involve the sex and autosomal chromosomes. It was reported that most infertile men with constitutional chromosomal abnormalities had $47, X X Y$ or Robertsonian, or reciprocal translocation of autosomes. It has been found that $13.7 \%$ of azoospermic men and $6 \%$ of oligozoospermic men have an abnormal karyotype. In the azoospermic group, sex chromosome abnormalities predominated, mainly 47, XXY. Van Assche et al. (5) reported that autosomal anomalies, such as Robertsonian and reciprocal translocations, were the most frequent karyotypic abnormalities in the oligozoospermic group. The present case, with balanced chromosomal translocation of chromosomes 6 and 7 , represents the first case of complex chromosome rearrangements in a man with normal sperm count (average $29.5 \times 10^{5}$ / cc). Gekas et al. (6) reported that the incidence of chromosome abnormality was $3.02 \%$ in patients with normal sperm concentrations.

Cytogenetic analyses are recommended for couples with recurrent miscarriages, when clinical data fail to clarify the cause, as in the present case (2). Approximately $50 \%$ of SABs result from genetic abnormalities (1). Balanced translocations were found in $0.6 \%$ of infertile couples, and $9.2 \%$ of infertile couples who experienced consecutive first-trimester abortions (2). Jacobs et al. (7) reported that the frequency of balanced chromosomal translocations in the general population was $0.3 \%$. They also reported that the incidence of balanced chromosomal translocations was $3.6 \%$ in couples who had had two or more SABs.

PGD has been used as an alternative to traditional prenatal diagnosis for couples who are at a high risk of transmitting a serious genetic disease or structural chromosomal abnormality to their offspring $(1,7)$. With developments in PGD, the detection of these structural chromosomal anomalies in couples having assisted reproductive treatment becomes very important. Kyu Lim et al. (8) performed PGD using FISH in 70 cycles of 49 couples with chromosomal translocations. The matured 
oocytes were fertilised by ICSI. They reported that after PGD, the spontaneous abortion rate was dramatically reduced from $95.8 \%(69 / 72)$ to $16.7 \%(3 / 18)$ in couples with translocations. We also referred the couple of the present case for ICSI and PGD.

Balanced chromosomal translocations frequently result in reproductive failure in infertile couples with a history of recurrent abortions. PGD should be performed in infertile couples with two or more SABs. PGD reduces spontaneous abortions in carrier couples.

Ethics Committee Approval: Ethics committee approval was received for this study.

Informed Consent: Written informed consent was obtained from the patient.

Peer-review: Externally peer-reviewed.

Author contributions: Concept - S.R., A.K.; Design - S.R.; Supervision - A.K.; Resource - ; Materials - S.R., T.A., A.K.; Data Collection\&/or Processing - S.R.; Analysis\&/or Interpretation - S.R., A.K., A.G.B.; Literature Search - T.A., E.E.; Writing - S.R., A.G.B.; Critical Reviews - S.R., A.G.B.

Conflict of Interest: No conflict of interest was declared by the authors.

Financial Disclosure: No financial disclosure was declared by the authors.

\section{References}

1. Cramer DW, Wise LA. The epidemiology of recurrent pregnancy loss. Semin Reprod Med 2000;18:331-9. [CrossRef]

2. Tunç E, Demirhan O, Demir C, Tastemir D. Cytogenetic study of recurrent miscarriages and their parents. Genetika 2007;43:545-52.

3. Moorhead PS, Nowell PC, Mellman WJ, Battips DM, Hungerford DA. Chromosomes preparations of leucocytes cultured from human peripheral blood. Exp Cell Res 1960;20:613-6. [CrossRef]

4. Martin RH, Hultén M. Chromosome complements in 695 sperm from three men heterozygous for reciprocal translocations, and a review of the literature. Hereditas 1993;118:165-75. [CrossRef]

5. Van Assche E, Bonduelle M, Tournaye H, Joris H, Verheyen G, Devroey $P$, et al. Cytogenetics of infertile men. Hum Reprod 1996;11:1-24. [CrossRef]

6. Gekas J, Thepot F, Turleau C, Siffroi JP, Dadoune JP, Briault S, et al. Chromosomal factors of infertility in candidate couples for ICSI:an equal risk of constitutional aberrations in women and men. Hum Reprod 2001;16:82-90. [CrossRef]

7. Jacobs PA, Frackiewicz A, Law P. Incidence and mutation rates of structural rearrangements of the autosomes in man. Ann Hum Genet 1972;35:301-19. [CrossRef]

8. Kyu Lim C, Hyun Jun J, Mi Min D, Lee HS, Young Kim J, Koong MK, et al. Efficacy and clinical outcome of preimplantation genetic diagnosis using FISH for couples of reciprocal and Robertsonian translocations:the Korean experience. Prenat Diagn 2004;24:556-61. [CrossRef] 